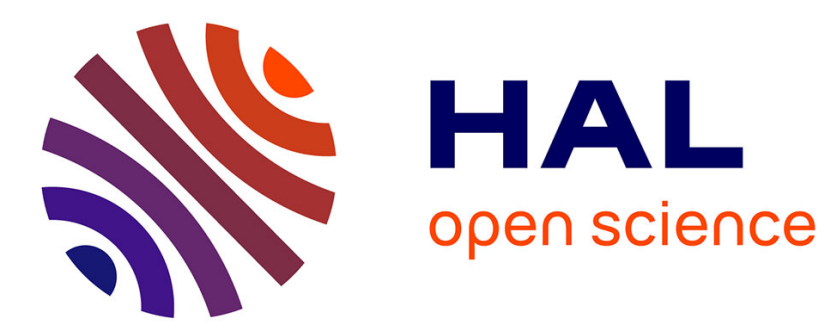

\title{
Spectral Rigidity in the Large Modal Overlap Regime: Beyond the Ericson-Schroeder Hypothesis
}

Olivier Legrand, Fabrice Mortessagne, Didier Sornette

\section{To cite this version:}

Olivier Legrand, Fabrice Mortessagne, Didier Sornette. Spectral Rigidity in the Large Modal Overlap Regime: Beyond the Ericson-Schroeder Hypothesis. Journal de Physique I, 1995, 5 (8), pp.1003-1010. 10.1051/jp1:1995179 . jpa-00247111

HAL Id: jpa-00247111

https://hal.science/jpa-00247111

Submitted on 1 Jan 1995

HAL is a multi-disciplinary open access archive for the deposit and dissemination of scientific research documents, whether they are published or not. The documents may come from teaching and research institutions in France or abroad, or from public or private research centers.
L'archive ouverte pluridisciplinaire $\mathbf{H A L}$, est destinée au dépôt et à la diffusion de documents scientifiques de niveau recherche, publiés ou non, émanant des établissements d'enseignement et de recherche français ou étrangers, des laboratoires publics ou privés. 
Classification

Physics Abstracts

$03.65 \mathrm{Sq}-05.45+\mathrm{b}$

\title{
Spectral Rigidity in the Large Modal Overlap Regime: Beyond the Ericson-Schroeder Hypothesis
}

\author{
Olivier Legrand, Fabrice Mortessagne and Didier Sornette \\ Laboratoire de Physique de la Matière Condensée( ${ }^{*}$ ), Université de Nice-Sophia Antipolis, Parc \\ Valrose, 06108 Nice Cedex 02, France \\ and \\ X-RS, Parc-Club - 28, rue Jean Rostand, 91893 Orsay Cedex, France \\ (Received 27 March 1995, accepted 4 May 1995)
}

\begin{abstract}
Spectral correlations for the total cross section in chaotic scattering or, alternatively, for the resonance density function in chaotic reverberant rooms, are studied within the frame of the random matrix theory. This framework allows us to develop a theory of spectral correlations in the large modal overlap regime which goes beyond the Ericson- Schrœeder result of Lorentzian autocorrelation functions. Spectral rigidity is shown to lead to a different autocorrelation function which is universal in the limit of large resonance overlap. Numerical evidence for this signature of spectral rigidity is given within the frame of a 2-dimensional chaotic billiard model of a reverberant room, for which level repulsion and spectral rigidity are known to be well described by the Gaussian Orthogonal Ensemble in the absence of absorption.
\end{abstract}

\section{Introduction}

Since the works of Ericson in the field of nuclear reactions [1] and of Schroeder for room acoustics [2] in the sixties, it is only recently that there appeared a renewal of interest in the statistical properties of either wave scattering through a chaotic region [3-5] or in the frequency response function of a reverberant room which displays chaotic ray dynamics [6]. In nuclear reactions, at high energies, many melastic channels open leading to an overlapping of many resonances associated to the intermediate states of the compound nucleus. In this situation, peaks occur in the cross section whose inverse mean width $\Gamma^{-1}$ corresponds to the average life time of the compound nucleus. The fluctuations of the partial cross sections have been assumed by Ericson to be dominated by the Gaussian random character of uncorrelated partial widths connecting the input or output states with the intermediate ones. This led him to establish the Lorentzian covariance of the cross section which is due to the exponential decay of the compound at the rate $\Gamma$. Using the same basic arguments, Schroeder established independently the same Lorentzian "frequency autocorrelation function" for the frequency response function

$\left({ }^{*}\right)$ CNRS URA 190 
between two points in a reverberant room where the sound energy exponentially decays due to absorption. Both assumed that the total widths (or decay rates) of the resonances do not fluctuate much around the average width $\Gamma$ and this can be justified by the fact that these total widths may be viewed as composed of many partial widths. Both also obtain results where the specific statistical properties of the positions of the resonances are irrelevant.

It is the aim of the present paper to show that the resonance fluctuations described by random-matrix theory (RMT) (e.g., Gaussian orthogonal ensemble (GOE) for the spectra of Hamiltonian systems with time-reversal symmetry) may prove to be of chief importance when evaluating energy correlations for total cross sections in chaotic scattering or, analogously, frequency correlations for properly spatially-averaged response functions in wave problems with absorption. In particular, in the case of moderate or large resonance overlap, the Lorentzian shape turns to be a poor approximation for the energy autocorrelation function of the mentioned quantities. A somewhat related approach was initiated by Smlansky who tried to put on a firm basis the relevance of RMT in quantum chaotic scattering by using Dyson's circular orthogonal ensemble for the scattering matrix [3].

\section{A Simple Model for the Spectral Fluctuations in the Large Modal Overlap Regime}

Following the simplifying assumptions made by Ericson [1], we write the total cross section for transitions from a given in-state as:

$$
\sigma^{\text {tot }}(E) \propto(1-\operatorname{Re} S)
$$

where $S=S^{(0)}+i \sum_{3} \frac{\left|\gamma_{\jmath}\right|^{2}}{E-E_{\jmath}}$, denoting by $E_{\jmath}=\operatorname{Re} E_{\jmath}-i \Gamma / 2$ the complex energies of the intermediate states and assuming that the resonance widths are all equal. The amplitudes $\left|\gamma_{j}\right|^{2}$ of the resonances should vary from resonance to resonance but also from a given in-state to another one. Therefore, in the following, we consider an average of $\sigma^{\text {tot }}$ over in-states that we still denote by $\sigma^{\text {tot }}$ and which reads:

$$
\sigma^{\text {tot }} \propto 1-\operatorname{Re} S^{(0)}-a \sum_{\jmath} \frac{\Gamma / 2}{\left(E-\operatorname{Re} E_{\jmath}\right)^{2}+\Gamma^{2} / 4}
$$

This expression is in close relation to the Wigner-Eisenbud time delay $\tau(E)$ or resonance density $[7,8]$ defined as:

$$
\tau(E)=-i \operatorname{Tr} S^{\dagger} \frac{\partial S}{\partial E}
$$

and which has the following pole decomposition:

$$
\tau(E)=\frac{1}{\pi} \sum_{\jmath} \frac{\Gamma / 2}{\left(E-\operatorname{Re} E_{\jmath}\right)^{2}+\Gamma^{2} / 4}
$$

A formally equivalent problem is met when studying the real part of the so-called input acoustic impedance used, for instance, in the field of reverberation room acoustics. This will be the physical problem we address through a numerical model described in the following section.

In the following, we assume that the rigidity of the complex compound (or of the reverberant room) spectrum (for the real part of the energies) is fairly well described by the GOE statistics. What are then the implications of the corresponding spectral fluctuations on the 
autocorrelation function of the fluctuating part of the resonance density $\left\langle\tau^{\mathrm{f}}(E+\epsilon) \tau^{\mathrm{f}}(E)\right\rangle$ where $\tau^{\mathrm{f}}=\tau-\langle\tau\rangle$ ? Denoting by $D$ the local mean spacing between adjacent resonances, $\langle\tau\rangle$ is then equal to $1 / D$. Now, using the normalised variables $x=E / D, x_{j}=E_{j} / D$ and $\gamma=\Gamma / D$, and defining the normalised resonance density function $n(x)=D \tau(E)$, one can write the power spectrum of $n^{\mathrm{f}}=n-1$ as the Fourier transform of its autocorrelation function $R(x)$ :

$$
\int_{0}^{\infty} \cos \lambda x R(x) \mathrm{d} x=\mathrm{e}^{-\gamma|\lambda|}[1-b(\lambda)],
$$

where $b(\lambda)$ is the two-level form factor defined as the Fourier transform of the two-level cluster function $Y_{2}(x)$ [10] (see the Appendix). For the GOE, the two-level form factor reads:

$$
b(\lambda)= \begin{cases}1-\frac{\lambda}{\pi}+\frac{\lambda}{2 \pi} \ln (1+\lambda / \pi) & \lambda \leq 2 \pi \\ -1+\frac{\lambda}{2 \pi} \ln \left(\frac{\lambda / \pi+1}{\lambda / \pi-1}\right) & \lambda \geq 2 \pi\end{cases}
$$

The evaluation of the autocorrelation $R(x)$ is easily achieved in the limiting case of a very large $\gamma$ value, which amounts to consider the large modal overlap regime. In the simple model we analyse, only this limiting regime makes sense since the approximate constancy of the decay parameter is assumed. This simplifying assumption is commonly used in the acoustic literature $[2,6,9]$ since it is claimed to be true in practice in the case of a diffuse sound field [2]. We postpone a tentative justification of this assumption within the frame of the model reverberant room discussed in the following section.

Due to the exponential factor in the r.h.s of equation (5), only the leading behavior of $1-b(\lambda) \sim \lambda$ for small values of $\lambda$ is to be retained. Note that, in this regime, the same leading behavior is obtained for other Gaussian ensembles (GUE or GSE) or superpositions of independent spectra [10] but not in the Poisson statistics (which is conjectured to prevail for spectra of classically regular systems) for which $Y_{2}(x)=0$. Thus, in the limit of large resonance overlap, no other information but spectral rigidity should be revealed by the autocorrelation which is found to be related to the derivative of the Lorentzian:

$$
R(x)=\frac{1}{\pi^{2} \gamma^{2}} \frac{1-(x / \gamma)^{2}}{\left(1+(x / \gamma)^{2}\right)^{2}}=\frac{D^{2}}{\pi^{2} \Gamma^{2}} \frac{1-(\epsilon / \Gamma)^{2}}{\left(1+(\epsilon / \Gamma)^{2}\right)^{2}}=R(\epsilon) .
$$

It should be stressed again that $R(x)$ would be a Lorentzian in the case of a Poisson sequence of resonances with no level repulsion.

Expression (7) of the autocorrelation coincides with the limiting form given by Eckhardt in the context of a semiclassical analysis of a scattering problem where the width $\Gamma$ is a classical escape rate [8]. A similar form has also been derived by Shushin and Wardlaw for a particular scattering off a surface of constant negative curvature [11]. In contrast, our derivation makes no assumption about an equivalent classical problem but addresses a general dissipative wave problem. As an extension of the theories of Ericson and Schrœder it relies on the validity of a universal statistical feature of the spectrum, namely the rigidity.

\section{The Numerical Model}

Here we provide numerical support for the above predictions within the frame of a $2 \mathrm{D}$ model of a reverberant room, namely a chaotic billiard where the wave equation is solved subject to absorbing boundary conditions. We use a method based on ray trajectories, introduced 
previously $[12,13]$, to calculate the temporal response to a brief pulse in a chaotic billiard shaped $2 \mathrm{D}$ room in which the pressure $p$ satisfies the standard wave equation [12]:

$$
-\frac{\partial^{2}}{\partial t^{2}} p(\mathbf{x}, t)+\nabla^{2} p(\mathbf{x}, t)=-F(\mathbf{x}, t)
$$

together with the uniform absorbing boundary condition

$$
\frac{\partial p}{\partial n}=-\beta \frac{\partial p}{\partial t}
$$

where $\partial / \partial n$ denotes the inward normal gradient at the boundary which plays the role of an absorbing wall with a specific admittance $\beta$. Our construction is based on a summation over families of rays which connect a source point to a finite size region around a measure point so that the response is evaluated on a coarse-grained scale which amounts to a spatial and temporal averaging at high frequencies. Accounting for the absorption is achieved by reducing the amplitude of each contribution by a factor $(\cos \theta-\beta) /(\cos \theta+\beta)$ for each reflection at the wall, $\theta$ being the angle of incidence with respect to the normal.

The time response in such a model room is computed for an isotropic point source which is a Dirac pulse in time. The enclosure used is a dissymmetrized Sinai billiard in which the dynamics of the ray trajectories is chaotic in the strongest sense. It is also well known (see for instance Ref. [10]) that the high-frequency spectrum of a membrane with the shape of a chaotic billiard exhibits statistical features (e.g., level repulsion or spectral rigidity) which are very well described by the GOE. By Fourier transforming the decaying response, we calculated the frequency autocorrelation function in a frequency range where the mean spacing between resonances is much smaller than the decay rate. Two types of response were considered. The first type of response corresponds to a measure point which is different from the source location. For a point source at $\mathbf{y}$ and a measure point at $\mathbf{x}$, the Fourier transform of the response (its real part in fact), or the transmission function, approximately reads [9]:

$$
T(\mathrm{x}, \mathrm{y} ; \omega) \propto \sum_{\jmath} \varphi_{3}(\mathrm{x}) \varphi_{3}(\mathrm{y}) \frac{\Gamma / 2}{\left(\omega-\omega_{\jmath}\right)^{2}+\Gamma^{2} / 4}
$$

In the last expression, $\varphi_{3}$ is the amplitude of the $j^{\text {th }}$ resonance mode of the wave equation subject to the boundary conditions given by equation (9).

At this stage, we show how, in the large modal overlap regime, the assumption of constant decay parameter may be justified. The argument goes as follows. In our model, absorption is uniformly distributed on the boundary. At high frequencies, the decay parameter is obtained through an average of the squared pressure on the boundary which essentially is equivalent to a multi-channel dissipative process involving a large number $N$ of independent channels scaling as $P / \lambda$ ( $P$ being the perimeter of the $2 D$ room and $\lambda$ the wavelength). If we assume a Gaussian distribution for the pressure (deduced from an ergodic conjecture due to Berry, see [13] and references therein) and that the average partial decay rates for each channel are equal, i.e., $\left\langle\Gamma_{\jmath}\right\rangle=\langle\Gamma\rangle / N$, then a $\chi^{2}$ distribution with $N$ degrees of freedom is obtained:

$$
P_{N}(\Gamma) \mathrm{d} \Gamma=\left(\frac{N \Gamma}{2\langle\Gamma\rangle}\right)^{N / 2} \frac{1}{\Gamma(N / 2)} \exp \left[-\frac{N \Gamma}{2\langle\Gamma\rangle}\right] \frac{\mathrm{d} \Gamma}{\Gamma}
$$

Thus as $N$ increases (at high frequencies) the variance decreases:

$$
\sigma_{\Gamma}^{2} \equiv\left\langle\Gamma^{2}\right\rangle-\langle\Gamma\rangle^{2}=\frac{2}{N}\langle\Gamma\rangle^{2} \propto \frac{\lambda}{P}\langle\Gamma\rangle^{2}
$$




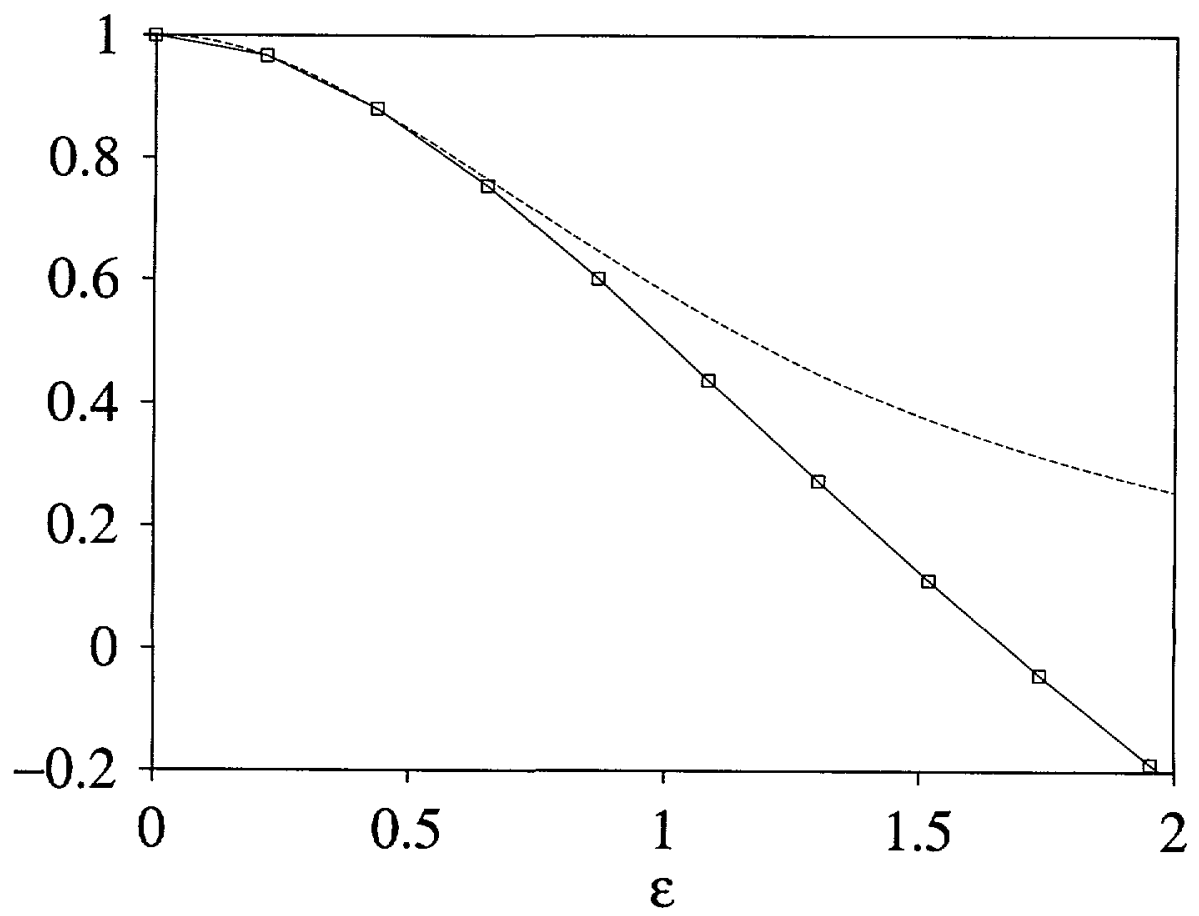

Fig. 1. - The normalized autocorrelation $R(\epsilon) / R(0)$ of the frequency response calculated in a twodimensional model of a reverberant room with the shape of a Sinai billiard is shown (squares) in the case of two uncorrelated source and measurement locations. The value of the decay rate $\Gamma \approx 1.17$ (deduced from an exponential fit of the temporal decay response) is substituted in the Lorentzian $\left[1+(\epsilon / \Gamma)^{2}\right]^{-1}$ which is plotted (dashed line) for comparison. Frequency averaging is obtained in a high frequency window whose width is around 50 decay rates. The corresponding frequency range is in the large modal overlap regime since the estimated value of the local mean spacing $D$ between adjacent levels is of the order of 0.015 .

An estimate for $\langle r\rangle$ can be obtained through Sabine's formula $\langle\Gamma\rangle=P \alpha / \pi S$ (S being the area of the $2 D$ room) where $\alpha$ is the equivalent absorption coefficient [13] the realistic values of which being of the order of, or smaller than, unity. Using the leading term of Weyl's law at high frequencies, the mean spacing $D$ is approximately $\lambda / S$. Therefore, the large modal overlap regime $\langle\Gamma\rangle / D \gg 1$ immediately implies that $\sigma_{\Gamma}$ be much smaller than $\langle\Gamma\rangle$ thus ensuring the approximate constancy of the decay parameter.

Here it should be noted that there exist two sources of fluctuations for expression (10). One is associated with the random character of the mode amplitudes $\varphi_{3}$, the other is related to the fluctuations of the spectrum (real parts of the elgenfrequencies). If only the randomness of the products $\varphi_{3}(\mathrm{x}) \varphi_{3}(\mathrm{y})$ is considered one recovers the theories of Ericson and Schrceder. Indeed, for spatially uncorrelated points $\mathbf{x}$ and $\mathbf{y}$, the frequency autocorrelation function $\langle T(\omega+\epsilon) T(\omega))$ is expected to be of the Lorentzian type $\left[1+(\epsilon / \Gamma)^{2}\right]^{-1}$, due to the fact that the fluctuations of $T(\omega)$ are dominated by those of the mode amplitudes, at least for sufficiently small values of $\epsilon$. In Figure 1 this prediction is partially validated by comparing it with the autocorrelation function obtained numerically for a value of the decay rate $\Gamma$ deduced directly from the early exponential decay of the time dependent response. On the contrary when the response is measured at the location of the source, expression (10) reduces to the so- 


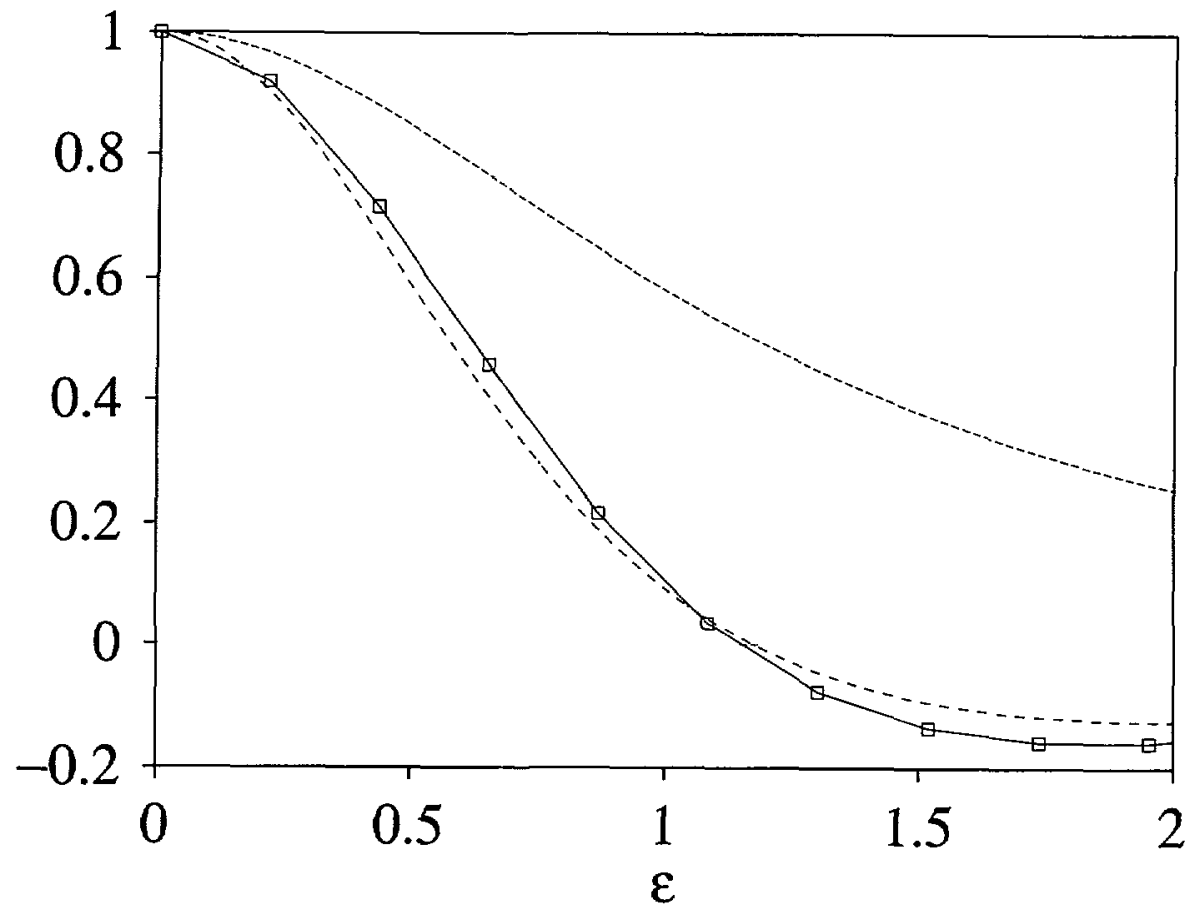

Fig. 2. - Same as in Figure 1 when measuring the response at the location of the source. For the sake of comparison, the prediction of formula (7) is shown (dashed-dotted line), as well as the Lorentzian (dashed line), both with $\Gamma \approx 1.17$. The agreement of the numerical result with our prediction with no adjustable parameter is excellent.

called input impedance of the source

$$
Z(\mathbf{x} ; \omega) \propto \sum_{j} \varphi_{\jmath}^{2}(\mathbf{x}) \frac{\Gamma / 2}{\left(\omega-\omega_{j}\right)^{2}+\Gamma^{2} / 4}
$$

When averaging this quantity over a number of different source locations, one is left with a quantity which essentially is the resonance density function given in equation (4)

$$
\overline{Z(\omega)} \propto \overline{\varphi^{2}} \sum_{j} \frac{\Gamma / 2}{\left(\omega-\omega_{3}\right)^{2}+\Gamma^{2} / 4} .
$$

In the last expression the overbar denotes the average over the source locations.

Therefore, the autocorrelation of the fluctuating part of equation (14) should bear the mark of the spectral rigidity as predicted in the preceding section. Indeed this is what we have found, as shown in Figure 2 where both the Lorentzian and our prediction given by expression (7) are compared to the numerically obtained autocorrelation. The Lorentzian cannot account for the shape and width of the autocorrelation in this case whereas our formula fits the numerical data very well. Again, it should be reminded that, for the normalized autocorrelation $R(\epsilon) / R(0)$, there is no adjustable parameter left since the value of $\Gamma$ is determined separately by a direct measurement. In both figures, the frequency averaging was performed in the same frequency window $\omega \in[95,150]$ where the mean spacing $D$ between adjacent resonances is of the order 
0.015 as estimated by using the leading term of Weyl's formula for the mean density of resonances. The absorption we used in both cases leads to a characteristic decay rate $\Gamma \approx 1.17$. For values of the frequency mismatch $\epsilon$ larger than a few widths (not shown in the figures), the frequency autocorrelation functions we observe clearly display non-universal oscllations (i.e., not predicted by assuming spectral rigidity as given by RMT). These should be related to the existence of non-universal features of the form factor, as emphasized by Berry [14], associated with the shortest periodic orbits of the billiard. Further investigation is needed to account for the non-universal long range behavior of the correlations probably through semiclassical calculations involving the least unstable periodic orbits.

\section{Conclusion}

We have provided simple arguments which show how spectral rigidity in complex spectra can be revealed in decaying processes even in the regime where the resonances are not resolved. Our approach is based on a simple model already used by Ericson and Schroder but includes one more essential ingredient, namely that the resonances have real parts that should be described by the random matrix theory. Using the two-point correlation function of the Gaussian ensembles of random matrices, we showed that the autocorrelation function of the resonance density should be different from the standard Lorentzian and established its expression in the case of large modal overlap. We then presented numerical support for our predictions by analysing the spectral content of the time response of a model 2D reverberant room. We surmise that this signature of spectral rigidity in dissipative complex wave systems has some close relation with the recent findings of Weaver concerning weak Anderson localization and enhanced coherent backscattering in reverberation rooms in the time domain [15].

\section{Appendix A}

Here, the normalised variables defined in the text are used. The normalised resonance density function is written as:

$$
n(x)=\frac{1}{\pi} \sum_{\jmath} \frac{\gamma / 2}{\left(x-x_{\jmath}\right)^{2}+\gamma^{2} / 4}=\frac{1}{\pi} \int \mathrm{d} x^{\prime} \frac{\gamma / 2}{\left(x-x^{\prime}\right)^{2}+\gamma^{2} / 4} z\left(x^{\prime}\right)
$$

where, for the sake of simplicity, the $x_{j}$ 's stand for the real parts, and the delta density function $z(x)$ is introduced:

$$
z(x)=\sum_{\jmath} \delta\left(x-x_{\jmath}\right)
$$

It is then known that, from the knowledge of the correlation functions of $z(x)$ which are deduced from the $n$-level distribution functions of the Gaussian ensemble, one may, in principle, find the correlation functions of the resonance density $n$. The two-level correlation function of $z$ reads $[16]$ :

$$
\langle z(x+X) z(x)\rangle=\delta(X)-Y_{2}(X)
$$

where $Y_{2}(x)$ is the two-level cluster function of the sequence of $x_{\jmath}$ 's which have unit density on the average.

For a given stationary random process $s(x)$, one defines the power spectrum $S(\lambda)$ as:

$$
S(s ; \lambda)=\int_{-\infty}^{+\infty} \mathrm{e}^{i \lambda x}\langle s(x+X) s(x)\rangle \mathrm{d} X .
$$


For the density function $z$, the power spectrum thus reads:

$$
S(z-<z>; \lambda)=1-b(\lambda)
$$

where the two-level form factor $b(\lambda)$ is the Fourier transform of $Y_{2}[10]$ :

$$
b(\lambda)=\int_{-\infty}^{+\infty} \mathrm{e}^{2 \lambda x} Y_{2}(x) \mathrm{d} x .
$$

Then the power spectrum of $n$ reads:

$$
\int_{-\infty}^{+\infty} \mathrm{e}^{2 \lambda X}\left[\langle n(X+x) n(x))-\langle n\rangle^{2}\right] \mathrm{d} X=\mathrm{e}^{-\gamma|\lambda|}(1-b(\lambda)) .
$$

\section{References}

[1] Ericson T., Phys. Rev. Lett. 5 (1960) 430-431; id., Ann. Phys. 23 (1963) 390-414.

[2] Schroeder M.R. and Kuttruff K.H., J. Acoust. Soc. Am. 34 (1962) 76-80; Schroeder M.R., J. Acoust. Soc. Am. 34 (1962) 1819-1823.

[3] Blümel R. and Smilansky U., Phys. Rev. Lett. 64 (1990) 241-244.

[4] Smilansky U., Chaos and Quantum Physics, M.-J. Giannonı, A. Voros and J. Zinn-Justin, Eds. (Elsevier, Amsterdam, 1991) pp 371-441.

[5] Doron E., Smilansky U. and Frenkel A., Phys. Rev. Lett. 65 (1990) 3072-3075.

[6] Weaver R.L., J. Sound Vib. 130 (1989) 487-491.

[7] Doron E. and Smilansky U., Phys. Rev. Lett. 68 (1992) 1255-1258.

[8] Eckhardt B., Chaos 3 (1993) 613-618.

[9] Davy J.L., J. Sound Vib. 77 (1981) 455-479.

[10] Bohigas O., Chaos and Quantum Physics, M.-J. Giannonı, A. Voros and J. Zinn-Justin, Eds. (Elsevier, Amsterdam, 1991) pp. 87-199.

[11] Shushin A. and Wardlaw A., J. Phys. A 25 (1992) 1503.

[12] Mortessagne F., Dynamıque et interférences géométriques dans les billards chaotiques. Application à l'acoustique des salles, Thèse de l'Université de Parıs 7 (1994).

[13] Mortessagne F., Legrand O. and Sornette D., Chaos 3 (1993) 529-541; Mortessagne F., Legrand O. and Sornette D. (preprint).

[14] Berry M.V., Chaos and Quantum Physics, M.-J. Giannoni, A. Voros and J. Zinn-Justin, Eds. (Elsevier, Amsterdam, 1991) pp. 251-303.

[15] Weaver R.L. and Burkhardt J., J. Acoust. Soc. Am. 96 (1994) 3186-3190.

[16] Brody T.A. et al., Rev. Mod. Phys. 53 (1981) 385-479. 